\title{
The Nuclear Equation of State
}

\author{
James M. Lattimer*i \\ Department of Physics \& Astronomy, Stony Brook University \\ E-mail: lattimer@astro.sunysb.edu
}

Recent observations from cooling neutron stars and of photospheric radius expansions in X-ray bursters are used to simultaneously estimate their masses and radii. Although the observational uncertainties for these sources are considerable, as a group they snugly constrain the mass-radius relation and the equation of state. The results of a Bayesian analysis using a parametrized equation of state are discussed. It is shown that the low-density equation of state is consistent with that obtained from recent estimates for pure neutron matter. Also, the deduced nuclear incompressibility is surprisingly compatible with nuclear systematics and experiment. The density dependence of the nuclear symmetry energy is predicted to be relatively small, leading to correspondingly small values for the predicted neutron skin thickness of lead and for the radii of $1.4 \mathrm{M}_{\odot}$ stars. The high-density equation of state stiffens, however, and the neutron star maximum mass, to $90 \%$ confidence, is predicted to be greater than 1.85 solar masses.

11th Symposium on Nuclei in the Cosmos, NIC XI

July 19-23, 2010

Heidelberg, Germany

\footnotetext{
*Speaker.

${ }^{\dagger}$ I appreciate funding from DOE under grant DE-AC02-87ER40317.
} 


\section{Introduction}

One of the outstanding problems in nuclear astrophysics concerns the equation of state (EOS) of dense matter, especially above the nuclear saturation density. Recently, substantial progress has been achieved in two directions: an improved theoretical understanding of the properties of pure neutron matter and accumulating observations of neutron star masses and radii. A detailed discussion of possible links between observables, neutron star structure and the EOS is contained in a recent review [1]. This contribution summarizes these developments, the details of which can be found in Refs. [2] and [3].

\section{General Theoretical Constraints}

It is worthwhile to summarize global constraints on neutron star structure from the fundamental theoretical perspectives of general relativity and causality. Assuming the pressure $p$ is causally constrained above an energy density $\varepsilon_{f}$ (i.e., $p \leq p_{f}+\varepsilon-\varepsilon_{f}$ ), one finds $M_{\max } \leq 4.2\left(\varepsilon_{s} / \varepsilon_{f}\right)^{1 / 2} \mathrm{M}_{\odot}[4]$, where $\varepsilon_{s}$ is the nuclear saturation energy density. Also, the compactness is limited to $R / M \geq$ $2.9 G / c^{2}$ [5]. It can be also shown that the largest well-measured mass sets an absolute upper limit to the density possible in any neutron star: $\rho_{c}<4.5 \times 10^{15}\left(\mathrm{M}_{\odot} / M\right)^{2} \mathrm{~g} \mathrm{~cm}^{-3}$ [7]. The highest possible spin frequency is $f \leq 1.08\left(M_{s p h} / \mathrm{M}_{\odot}\right)^{1 / 2}\left(10 \mathrm{~km} / R_{s p h}\right)^{3 / 2} \mathrm{kHz}[6]$. The most rapidly rotating

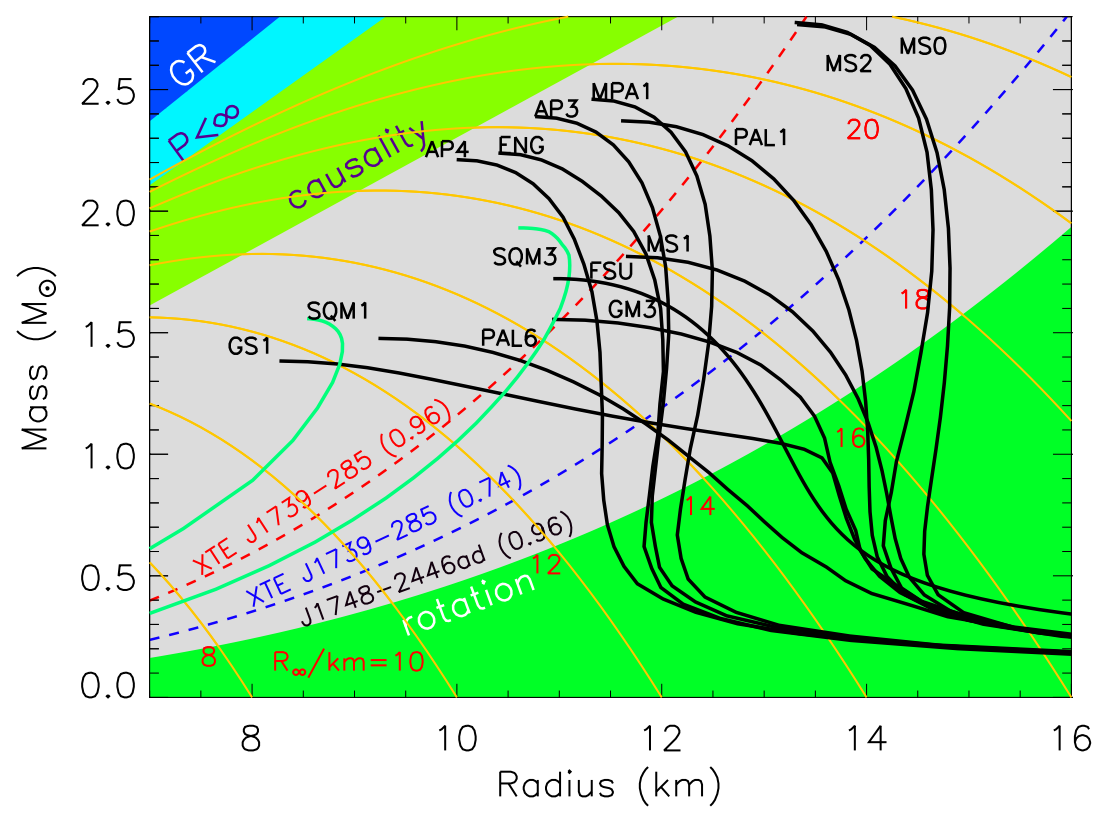

Figure 1: Mass-radius diagram with regions excluded by general relativity and causality in upper left corner. Baryonic (solid black) and strange quark (solid green) $M-R$ curves, as labelled in Ref. [8], are displayed. Lighter curves (solid orange) are $R_{\infty}=R / \sqrt{1-2 G M / R c^{2}}$ contours. The $716 \mathrm{~Hz}$ pulsar [9] mass-shedding limit is indicated by the gray-green boundary through which valid EOSs should enter. The red dashed line is a corresponding limit if the unconfirmed $1122 \mathrm{~Hz}$ spin frequency of XTE J1739-285 is valid [10].

millisecond pulsar is the object PSR J1748-2446ad, with a frequency of $716 \mathrm{~Hz}[9]$. This object 
spins rapidly enough to effectively constrain neutron star radii, especially if the star's mass (which is unknown) is in the typical pulsar range 1.2-1.4 $\mathrm{M}_{\odot}$, as shown in Fig. 1. A recent claim[10] of an even higher spin frequency, $1122 \mathrm{~Hz}$, from six type I X-ray bursts from the neutron star X-ray transient XTE J1739-285 has not been reproduced, however.

\section{Neutron Star Masses}

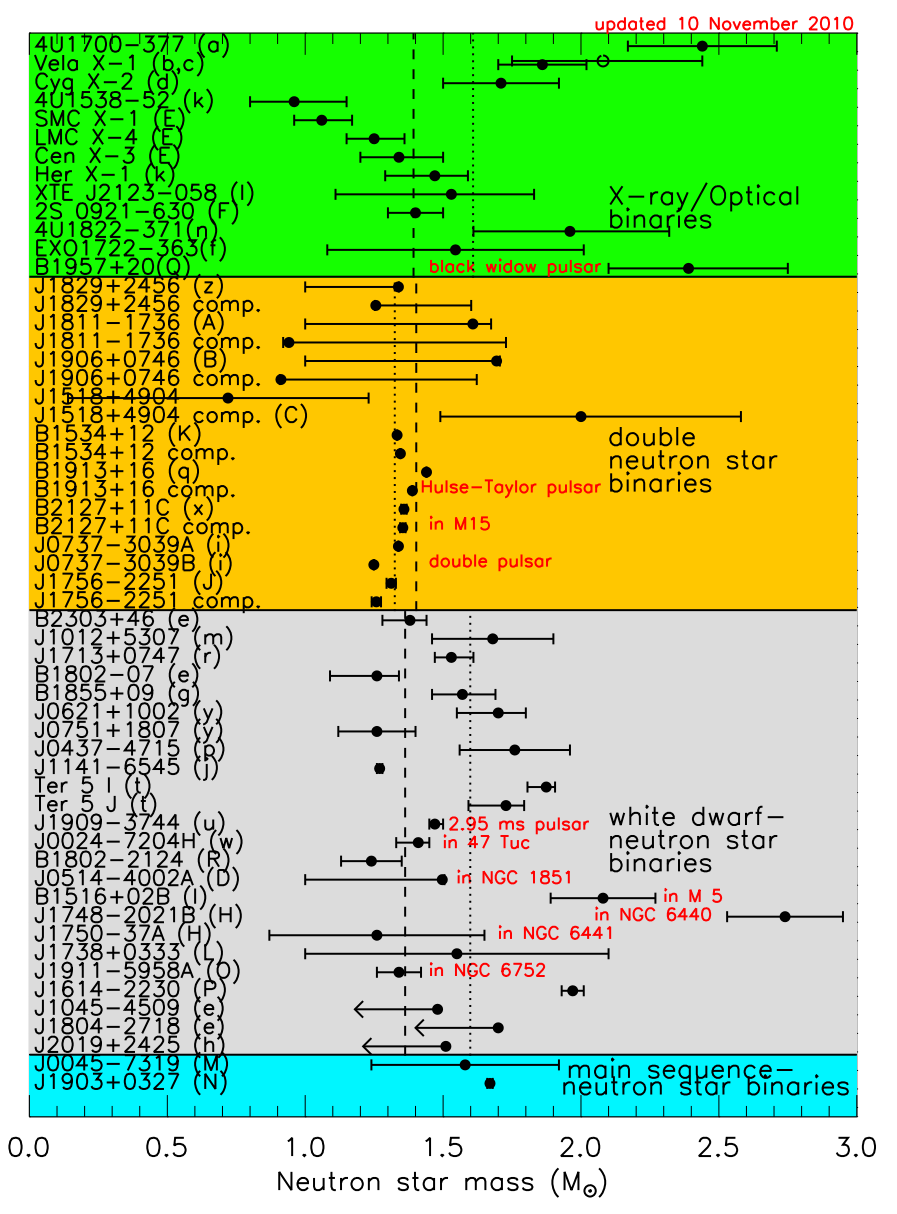

Figure 2: Neutron star mass measurements with 1- $\sigma$ uncertainties. Uppermost (green) region is for X-ray binaries, lowermost regions are for pulsar timing measurements. Dotted lines in each region indicate simple mass averages, dashed lines indicate weighted averages.

Our knowledge of neutron star masses is summarized in Fig. 2. The uppermost of the four regions contains mass estimates from X-ray binary sources, which are characterized by relatively large systematic errors. As Fig. 2 shows, the weighted mean of all X-ray binary masses is about $1.38 \mathrm{M}_{\odot}$, although a few sources, especially Vela X-1 [11], imply relatively large masses.

The lower three regions are mass measurements from binary radio pulsars, which have lower systematic uncertainties and hence higher potential accuracies. Even so, the two "high-mass" pulsars in the globular clusters M 5 (B1516+02B) and NGC 6440 (J1748-2021B) do not have measured inclinations [12] and should not be considered reliable. However, the inclination for the system J1903-0327 has been measured which results in a mass of $1.67 \pm 0.01 \mathrm{M}_{\odot}$ [13]. More 
striking is the large Shapiro delay measurement for the system PSR J1614-2230 [?] resulting in the mass $1.97 \pm 0.04 \mathrm{M}_{\odot}$ which now serves as the minimum maximum neutron star mass. Such a limit places severe constraints on the properties and composition of super-nuclear matter.

\section{Thermal Emission From Neutron Stars}

Following their birth, neutron stars cool via both neutrino and photon radiation. After about 50 seconds, the star becomes transparent to neutrinos which escape without scattering within the star. The high thermal conductivity of the core guarantees that it is nearly isothermal, and neutrino emission cools it more efficiently than the crust. The star is now only visible due to photon emission from its surface. The crust eventually becomes isothermal with the core but only after tens of years [14] corresponding to its thermal timescale. Dimensionally, the crust cooling time scales as $\Delta^{2} C_{V}\left(1-2 G M / R c^{2}\right)^{-3 / 2} / \dot{\varepsilon}$, where $\Delta$ is the crust thickness, $C_{V}$ is the crustal specific heat and $\dot{\varepsilon}$ is the neutrino emissivity of core material. The cooling time is possibly observable either following neutron star birth from a supernova remnant, or following crustal reheating during accretion (as an $\mathrm{X}$-ray transient). The crust thickness is a function of the star's mass, radius and core-crust transition density [1].

For the first million years after birth, neutrino emission from the neutron star dominates surface photon fluxes but will be too small to observe. The star will be visible as an X-ray (and, if the star is near enough, as an optical) source. Several such cooling neutron stars are observed. To zeroth order, thermal emission from neutron stars is similar to a blackbody, so measures of the integrated flux and the temperature yield an estimate of the angular diameter $R / D$ where $D$ is the star's distance. However, the flux is redshifted twice and the temperature once, so the inferred radius is actually $R_{\infty}=R / \sqrt{1-2 G M / R c^{2}}$, known as the radiation radius. Principal uncertainties in extracting this quantity include

- Distance uncertainties $\left(R_{\infty} \propto D\right)$

- Interstellar H absorption (most hard UV and an appreciable fraction of X-rays are absorbed between the star and the Earth)

- Atmospheric composition and magnetic field strength

The best chances of an accurate measurement are either from

- nearby isolated neutron stars, for which parallax distances are available, but which have unknown atmospheric compositions and magnetic field strengths, or

- quiescent X-ray binaries with reliable distances and, due to recent accretion, low magnetic fields and, almost certainly, H-dominated atmospheres.

The best-studied isolated neutron star is RX J1856-3754 [15], with a parallax distance of about 120 pc, recently confirmed by [16]. Unfortunately no adequate heavy-element, highly-magnetized atmospheric model exists. A two-temperature blackbody fits the overall spectrum well, and suggests $R_{\infty} / D \simeq 0.13 \pm 0.01 \mathrm{~km} / \mathrm{kpc}$ [17]. Ref. [18] proposed a highly-magnetized, condensed hydrogen, atmosphere with a similar angular diameter, but this requires a finely-tuned, trace, amount of hydrogen in the atmosphere whose origin is unclear. 

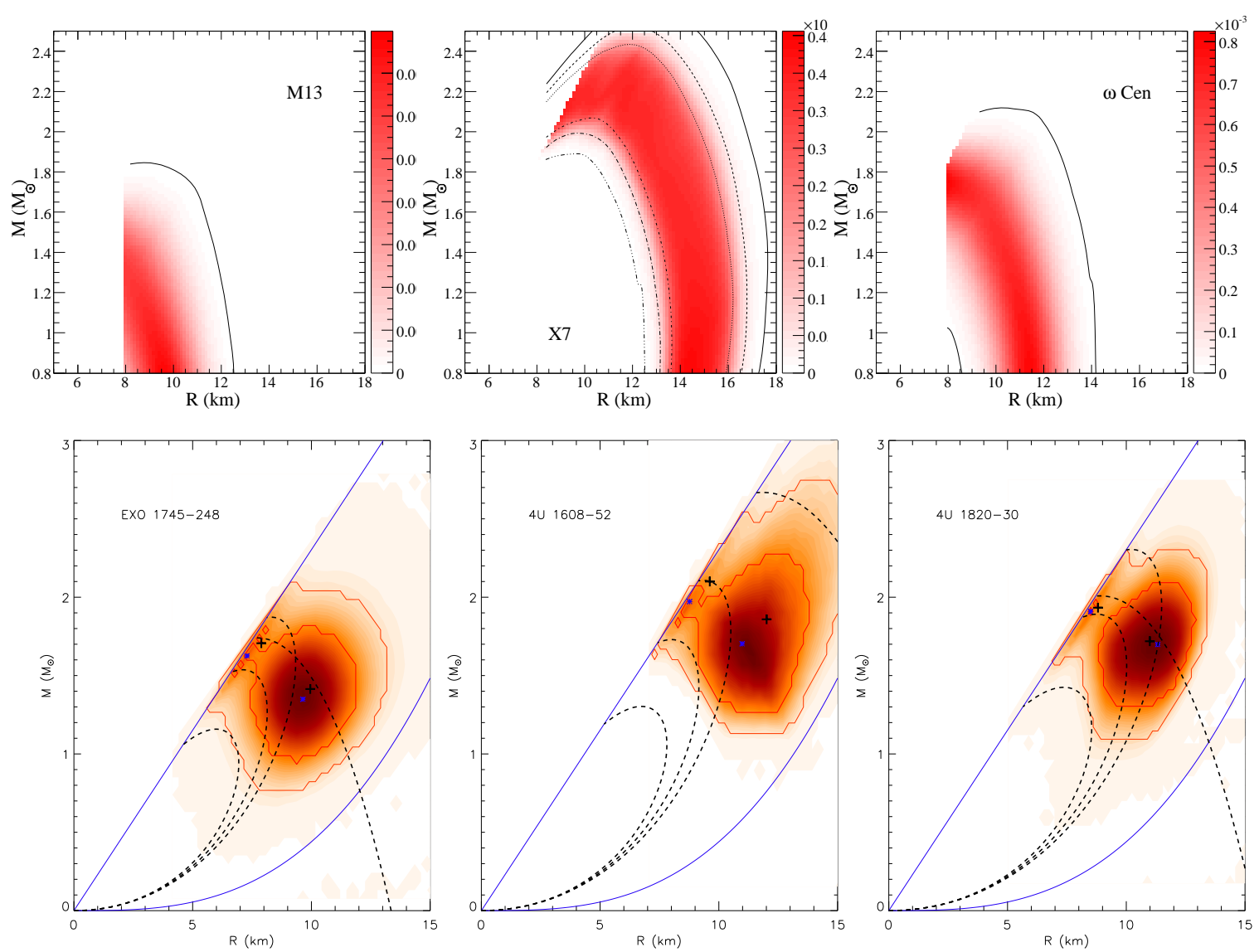

Figure 3: Mass and radius probability regions. Top: Thermally-emitting neutron stars. Solid curves show $99 \%$ probability extremes for M13 and $\omega$ Cen; the 6 curves for X7 outline $68 \%, 90 \%$ and $99 \%$ confidence regions. Figure from Ref. [3]. Bottom: Photospheric radius expansion burst sources. The causality limit $R=2.94 G M / c^{2}$ is the diagonal line. $68 \%$ and $95 \%$ contours are indicated by the two irregular contours encompassing the shaded regions. Also shown are lines denoting central values of $\gamma$ and $R_{\infty}$.

Many neutron stars are transients for which the accretion of matter proceeds intermittently, with episodes of accretion separated by long periods of quiescence. When the neutron star accretes, compression of matter in the crust induces nuclear reactions [19] that release heat. When the accretion ceases, the heated crust cools, resulting in an observable thermal luminosity [20]. Because the timescale for heavier nuclei to sink below the photosphere is short and these systems show no evidence, such as pulsations or cyclotron spectral features, for a significant magnetic field, the spectra can be fitted with well-understood unmagnetized hydrogen atmosphere spectra which can be used to reliably infer an apparent angular emitting area, and, possibly, the surface gravity [21]. Results [21, 22] for sources in 3 globular clusters are summarized in Fig. 3.

\section{Photospheric Radius Expansion Bursts}

Type I X-ray bursts are the result of thermally unstable helium (or in some cases, hydrogen) ignition in the accreted envelope of a neutron star [23]. The ignition generates a thermonuclear 
explosion that is observed as an X-ray burst with a rapid rise time $(\sim 1 \mathrm{~s})$ followed by cooling decay ( $\sim 10-100$ s). If the burst is sufficiently luminous, radiation pressure drives the photosphere outwards to larger radii, and the flux at the photosphere approaches (to within a few percent) the Eddington value before decreasing. The observed temperature initially decreases, then also increases before decreasing again. During this time, the normalized area

$$
A=\frac{F_{\infty}}{T_{\text {color }}^{4}}=f_{c}^{-4}\left(\frac{R_{\infty}}{D}\right)^{2}
$$

increases to a maximum value and then decreases as $T_{\text {color }}$ increases. The color correction factor $f_{c}=T_{b b, \infty} / T_{\text {color }}$ takes into account the neutron star atmosphere which modifies the usual blackbody relation. The point at which $T_{\text {color }}$ reaches a maximum (and the normalized area typically stops decreasing and becomes constant) is thought to be when the photosphere "touches down" near the stellar radius $R$. The flux at this time is thought to be the Eddington flux

$$
F_{E d d}=\frac{c G M}{\kappa D^{2}} \sqrt{1-\beta_{p h}}
$$

where $\kappa$ is the opacity, $\beta_{p h}=2 G M /\left(R_{p h} c^{2}\right)$ and $R_{p h}$ is the effective photospheric radius. Dynamical models indicate that $R_{p h}$ might be considerably larger than $R$ when the maximum flux is observed. We define, with $\beta=2 G M /\left(R c^{2}\right)$,

$$
\begin{gathered}
\alpha \equiv \frac{F_{E d d}}{\sqrt{A}} \frac{\kappa D}{c^{3} f_{c}^{2}}=\beta \sqrt{1-2 \beta} \sqrt{1-2 \beta_{p h}}, \\
\gamma \equiv \frac{A c^{3} f_{c}^{4}}{F_{E d d} \kappa}=\frac{R}{\beta(1-2 \beta) \sqrt{1-2 \beta_{p h}}} .
\end{gathered}
$$

Note that $\alpha \gamma=R_{\infty} . M$ and $R$ can be determined if $\alpha$ and $\gamma$ are known. If $R_{p h}=R, \alpha=\beta(1-\beta)$ which has real-valued solutions if $\alpha \leq 1 / 8$. However, if $R_{p h}>>R, \alpha=\beta \sqrt{1-2 \beta}$ and real-valued solutions exist if $\alpha \leq 3^{-3 / 2} \simeq 0.192$.

Ozel and collaborators (see, e.g., Ref. [24]) have studied the bursters EXO 1745-248, 4U 1608-522 and $4 \mathrm{U} 1820-30$ and determined $\alpha \simeq 0.13 \pm 0.02,0.18 \pm 0.06$ and $0.17 \pm 0.02$ for each. In addition, an analysis of the burster $4 \mathrm{U} 1724-307$ [25] implies a value $\alpha \simeq 0.15 \pm 0.04$. Thus, no real-valued solutions exist for any of them if $R_{p h}=R$ (note that the curves of fixed $\gamma$ and $R_{\infty}$ in Fig. 3 do not intersect). Monte Carlo sampling of $F_{E d d}, A, D, f_{c}$ and $\kappa$ within their estimated errors results in trial acceptance rates of only $0.13,0.0002,2 \cdot 10^{-8}$ and 0.65 . Extremely small acceptance rates generally indicate serious problems for a model's consistency. It also forces the accepted trials to have mean values of $\alpha$ near $1 / 8$ and $\gamma$ near $110 \mathrm{~km}$ with little variance irrespective of estimates for the observables. This results in estimates for $M$ and $R$ with very small error ranges; typically $1-\sigma$ errors are less than $5 \%$ [24]. If the model is generalized, however, to allow for the possibility that $R_{p h} \geq R$, the Monte Carlo acceptance rates increase dramatically [3], but predicted error ranges for $M$ and $R$ also increase, resulting in 1- $\sigma$ errors of order 15\%, as shown in Fig. 3. The most probable radii are approximately $1 \mathrm{~km}$ larger than Ref. [24] determined.

\section{Equation of State From Masses and Radii}

If one assumes a parametrized form for the EOS, values of the parameters can be obtained from a Bayesian analysis of the mass and radius probability distributions, using Markov Chain 
Monte Carlo integration techniques, as described in Ref. [3]. We divide the EOS into four density regimes. The region below the transition energy density $\varepsilon_{\text {trans }} \approx \varepsilon_{0} / 2$ is the crust, for which we use a standard EOS.. For $\varepsilon_{\text {trans }}<\varepsilon<\varepsilon_{1}$, we use a schematic expression representing charge-neutral uniform baryonic matter in beta equilibrium that is compatible with laboratory data:

$$
\frac{\varepsilon}{n_{B}}=m_{B}+B+\frac{K}{18}(u-1)^{2}+\frac{K^{\prime}}{162}(u-1)^{3}+(1-2 x)^{2}\left[S_{k} u^{2 / 3}+S_{p} u^{\gamma}\right]+e_{e}
$$

where $n_{B}$ is the baryon number density, $m_{B}$ is the baryon mass, $u=n_{B} / n_{s}$, and $x$ is the proton
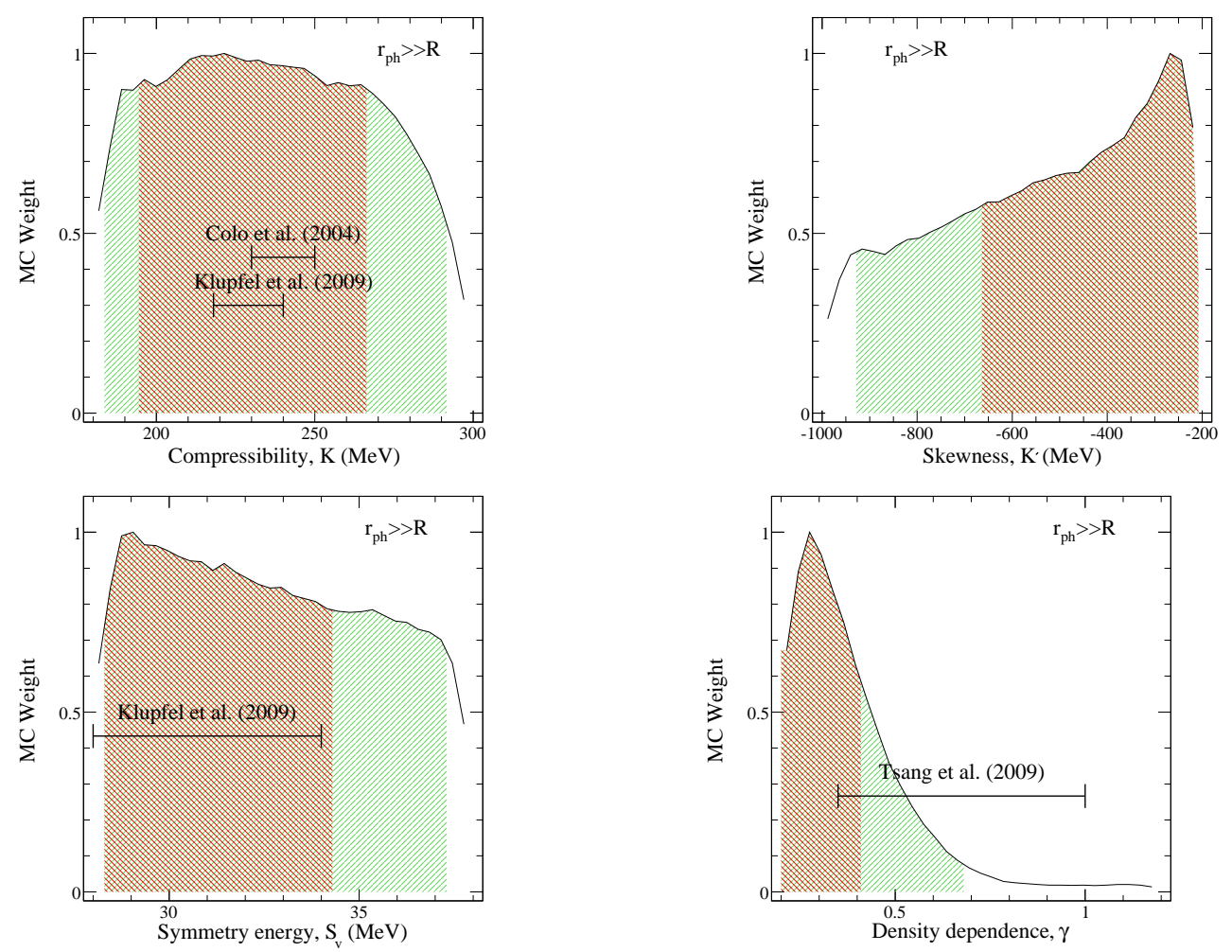

Figure 4: Histograms of the probability distributions from the Bayesian analysis for parameters of nuclear matter, defined in Eq. (6.1). The two shaded regions correspond to the 68\% and 95\% regions. Experimental estimates are indicated by horizontal error bars [26].

(electron) fraction. The saturation number density, $n_{s}=0.16 \mathrm{fm}^{-3}$, the binding energy of saturated nuclear matter, $B=16 \mathrm{MeV}$, and the kinetic part of the symmetry energy, $S_{k}=17 \mathrm{MeV}$ are held fixed. The compressibility $K$, the skewness $K^{\prime}$, the bulk symmetry energy parameter, $S_{v} \equiv S_{p}+S_{k}$ (where $S_{p}$ is the potential part of the symmetry energy), and the density dependence of the potential part of the symmetry energy, $\gamma$, are parameters. $e_{e}$ is the electron energy per baryon. The value of $x$ for each density is determined by the condition of beta equilibrium, $\partial \varepsilon / \partial x=0$. Above the density $\varepsilon_{1}$, two polytropic relations with exponents $\gamma_{1}$ and $\gamma_{2}$, separated at $\varepsilon_{2}$, are used. We did not include correlations among parameters, even though some are known to exist, e.g., nuclear mass formula fits [27] relate $S_{v}$ and $\gamma$ (or, equivalently, $S_{v}$ and $S_{s}$, the bulk and surface symmetry parameters). We restricted parameters to satisfy causality and the maximum mass limit of $1.66 \mathrm{M}_{\odot}$. 

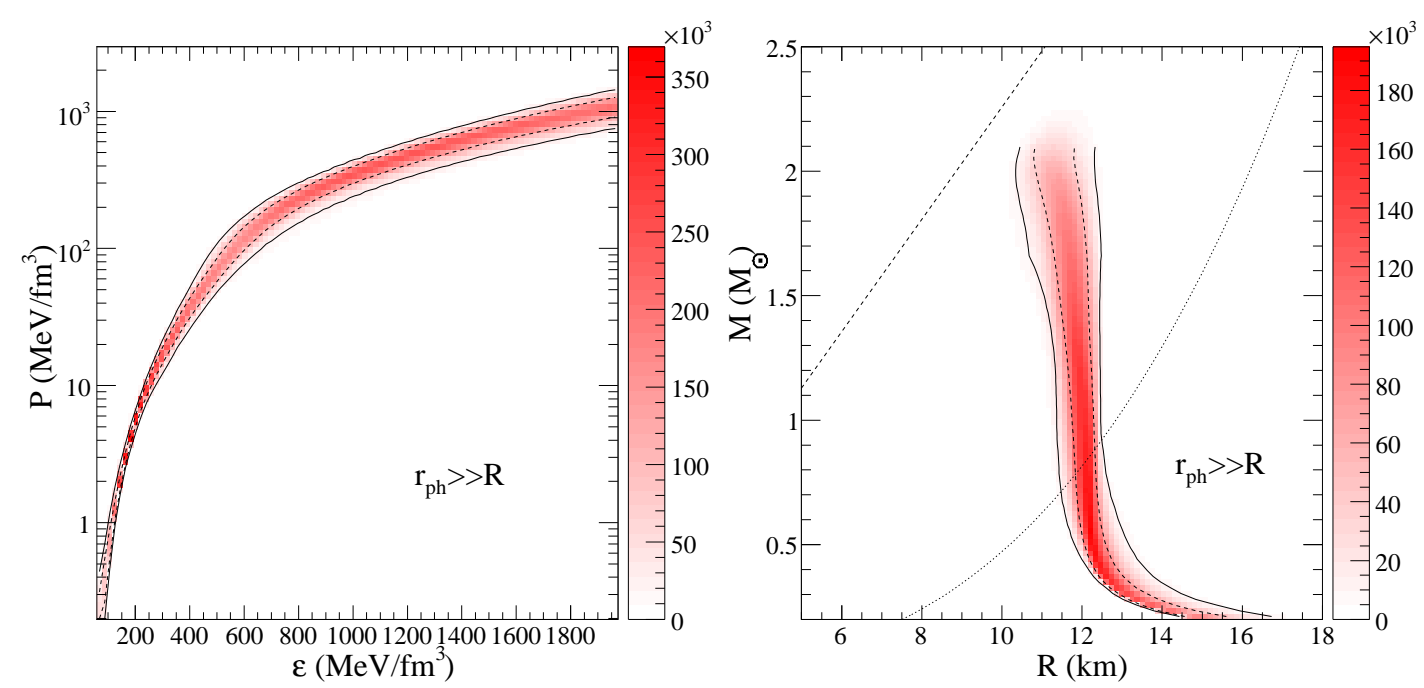

Figure 5: Left: Probability distributions for the pressure as a function of energy density using the mass and radius estimates from Fig. 3. Right: The probability distributions for the mass-radius relation.

It is notable that the four nuclear matter parameters are determined to have values in complete compatibility with experimental estimates, as shown in Fig. 4. Moreover, relatively small values of $\gamma$ are strongly indicated. This implies that both the nuclear symmetry energy and the pressure of neutron star matter are relatively soft, which will also lead to predicted small neutron star radii [8]. The most probable pressures, as a function of energy density, are displayed in the top panel of Fig. 5. Assuming that all neutron stars obey the same mass-radius relation, the Bayesian analysis permits its estimation and this is shown in the bottom panel of Fig. 5. Indeed, it is seen that, for masses of order $1.4 \mathrm{M}_{\odot}$, predicted radii are of order $11-12 \mathrm{~km}$.

Somewhat surprisingly, Fig. 5 also indicates that the neutron star maximum mass is relatively large; the Bayesian analysis indicates it is $2.05 \pm 0.11 \mathrm{M}_{\odot}$. This result, however, is quite sensitive to how photospheric radius expansion bursts are modeled; if one assumes $R_{p h}=R$, the predicted maximum mass distribution peaks below $1.7 \mathrm{M}_{\odot}$.

It is important to remember that these estimates are made on the basis of relatively poor radiusmass information from only 7 neutron stars. These limits should get tighter as additional sources become available. Nevertheless, more sophisticated modeling of photospheric radius expansion bursts and neutron star atmospheres will also be required for additional progress.

\section{Compatibility with Pure Neutron Matter}

Studies based on microscopic calculations of neutron matter using chiral low-momentum twonucleon and three-nucleon interactions allow the estimation of theoretical uncertainties due to neglected many-body forces and from an incomplete many-body calculation [28]. The largest uncertainty comes from the couplings that determine the leading order three-body forces in chiral effective field theory. At low densities, of order 1/10 the saturation density, the results [2] are consistent with resonant Fermi gases including effective range contributions [29]. If the symmetry energy is parametrized as in 6.1, the parameters $S_{p}$ and $\gamma$ can be estimated from the energy $E_{N}$ and 
pressure $P_{N}$ of neutron matter at the saturation density $n_{s}$ :

$$
S_{p}=E_{N}-S_{k}+B \simeq 15.3 \pm 2.1 \mathrm{MeV}, \quad \gamma=\frac{P_{N} / n_{s}-2\left(E_{N}+B\right) / 3}{S_{p}} \simeq 0.28 \pm 0.29 .
$$

Fig. 6 shows that the beta equilibrium neutron star matter from the pure neutron matter results are remarkably consistent with the astrophysical estimates shown in Fig. 5.

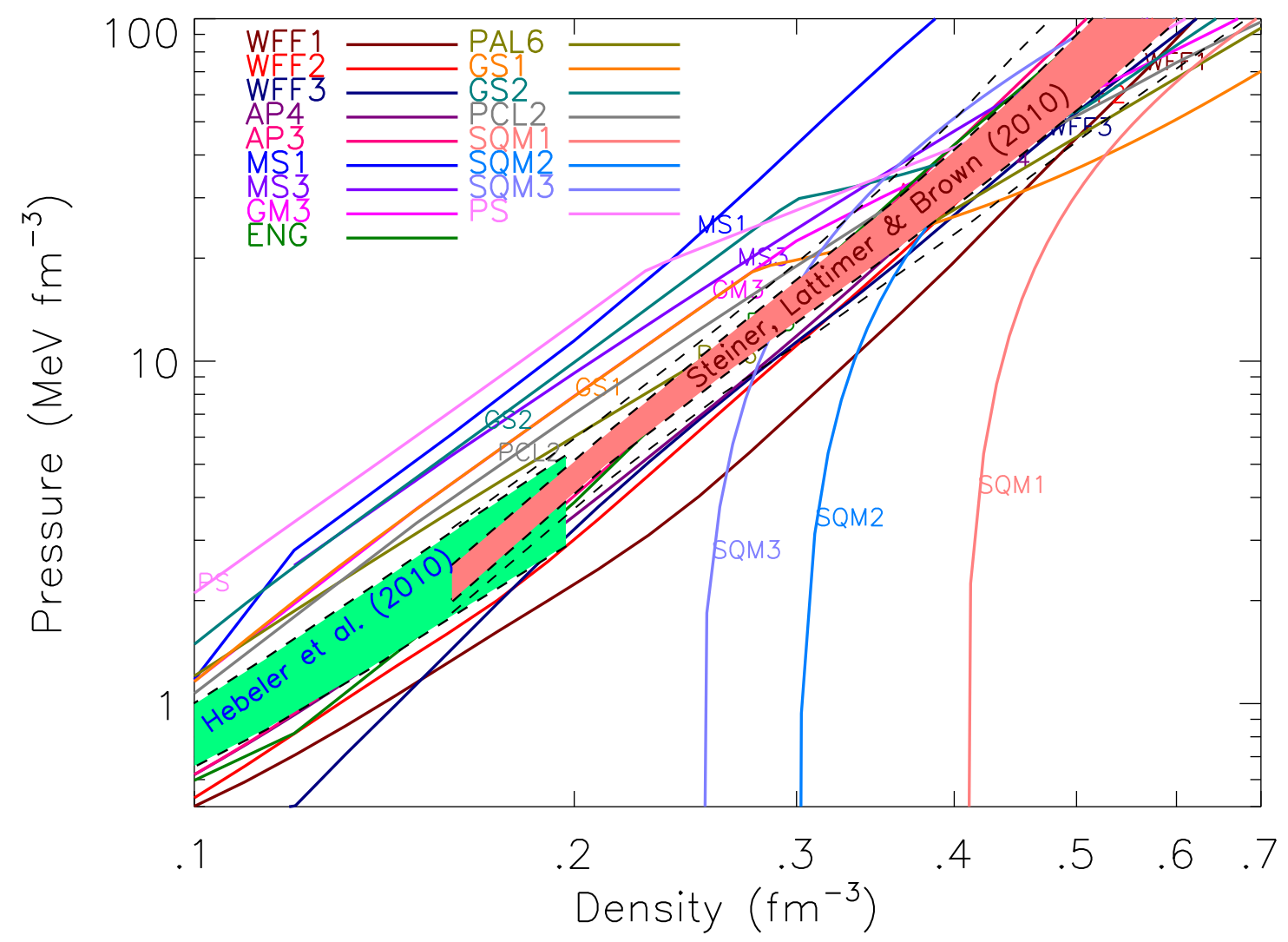

Figure 6: Neutron star matter pressure from neutron matter calculations of Ref. [2] (lower shaded region) is shown in comparison to astrophysical estimates from Ref. [3] (upper shaded region, 1- and 2- $\sigma$ contours shown as dashed lines). Other popular EOSs are described in Ref. [8].

\section{References}

[1] J. M. Lattimer and M. Prakash, Phys. Rep. 442, 109 (2007).

[2] K. Hebeler, J. M. Lattimer, C. J. Pethick and A. Schwenk, Phys. Rev. Lett. 105, 161102 (2010).

[3] A. W. Steiner, J. M. Lattimer and E. F. Brown, Ap. J. 722, 33 (2010).

[4] C. E. Rhoades Jr. and R. Ruffini, Phys. Rev. Lett. 32, 324 (1974); J. B. Hartle and A. G. Sabbadini, Ap. J. 213, 831 (1977).

[5] L. Lindblom, Ap. J. 278, 364 (1984); N. K. Glendenning, Phys. Rev. D46, 4161 (1990); S. Koranda, N. Stergioulas and J. L. Friedman, Ap. J. 488799 (1997).

[6] J. M. Lattimer and M. Prakash, Phys. Rev. Lett. 94, 1101 (2005). 
[7] J. M. Lattimer and M. Prakash, Science, 304, 536 (2004); P. Haensel, J. L. Zdunik, M. Bejger and J. M. Lattimer, A\& A, 502, 605 (2009).

[8] J. M. Lattimer and M. Prakash, Ap. J. 550, 426 (2001).

[9] J. W. T. Hessels, S. W. Ransom, I. H. Stairs, P. C. C. Freire, V. M. Kaspi and F. Camilo, Science, 311, 1901 (2006).

[10] P. Kaaret, P., Z. Prieskorn, J. J. M. In't Zand, S. Brandt, N. Lund, S. Mereghetti, D. Gotz, E. Kuulkers and J. .A. Tomsick, Ap. J. 657, L97 (2007).

[11] O. Barziv, L. Karper, M. H. van Kerkwijk, J. H. Telging and J. van Paradijs, A\& A, 377, 925 (2001); H. Quaintrell, A. J. Norton, T. D. C. Ash, P. Roche, B. Willems, T. R. Bedding, I. K. Baldry and R. P. Fender, A\& A, 401, 303 (2003).

[12] P. C. C. Freire, J. W. T. Hessels, S. M. Ransom, I. H. Stairs, S. Begin and A. Wolszczan, in 40 Year of Pulsars: Millisecond Pulsars, Magnetars, and More. AIP Conf. Proc., 983, 459 (2008).

[13] P. C. C. Freire, in Neutron Stars and Gamma Ray Bursts: Recent Developments and Future Directions, arXiv:0907.3219(2009).

[14] P. B. Demorest, T. Pennucci, S. M. Ransom, M. S. E. Roberts and J. W. T. Hessles, Nature 467, 1081 (2010).

[15] J. M. Lattimer, K. A. van Riper, M. Prakash and M. Prakash, Ap. J. 425, 802 (1994).

[16] F. M. Walter, S. Wolk, and R. Neuhäuser, Nature 379, ,233 (1996).

[17] F. M. Walter,T. Eisenbeiß, J. M. Lattimer, B. Kim, B., V. Hambaryan and R. Neuhäuser, Ap. J. 724, 669 (2010).

[18] J. A. Pons, F. M. Walter, F. M., J. M. Lattimer, M. Prakash, R. Neuhauser and P. An, Ap. J. 564 ,981 (2002).

[19] W. C. T. Ho, D. L. Kaplan, P. Chang, M. van Adelsberg and A. Y. Potekhin, Mon. Not. Roy. Ast. Soc. 375, 821 (2007).

[20] P. Haensel and J. L. Zdunik, A\& A, 227, ,431 (1990).

[21] E. F. Brown, L. Bildsten and R. E. Rutledge, Ap. J. 504 ,L95 (1998).

[22] C. O. Heinke, G. B. Rybicki, R. Narayan and J. L. Grindlay, Ap. J. 644 , 1090 (2006).

[23] N. A. Webb and D. Barret, Ap. J. 671, 727 (2007).

[24] T. J. Strohmayer and L. Bildsten, in Compact Stellar X-ray Sources, ed. W.H.G. Lewin and M. van der Klis (Cambridge, Boston, 2004) astro-ph/0301544.

[25] F. Özel, G. Baym and T. Güver, Phys. Rev. D. 82, 101301 (2010).

[26] V. Suleimanov, J. Poutanen, M. Revnivstev, and K. and Werner, arXiv:1004.4871 (2010).

[27] G. Colo, N. Van Giai, J. Meyer, K. Bennaceur and P. Bonche, Phys. Rev. C70, 024307 (,2004);

P. Klupfel, P.-G. Reinhard, T. J. Burvenich and J. A. Maruhn, Phys. Rev. C79, 034310 (2009);

M. B. Tsang, Y. Zhang, P. Danielewicz, M. Famiano, Z. Li, W. G. Lynch, and A. W. Steiner, Phys. Rev. Lett. 102, 122701 (2009).

[28] A. W. Steiner, M. Prakash, J. M. Lattimer and P. J. Ellis, Phys. Rep. 411, 325 (2005).

[29] K. Hebler and A. Schwenk, Phys. Rev. C82, 014314 (2020).

[30] A. Schwenk and C. J. Pethick, Phys. Rev. Lett. 95, 160401 (2005); A. Gezerlis and J. Carlson, Phys. Rev. C77, 032801 (2008). 